\title{
Observation of Interference between Two Distinct Autoionizing States in Dissociative Photoionization of $\mathbf{H}_{2}$
}

\author{
T. J. Reddish, ${ }^{1}$ A. Padmanabhan, ${ }^{1}$ M. A. MacDonald, ${ }^{2}$ L. Zuin, ${ }^{2}$ J. Fernández, ${ }^{3}$ A. Palacios, ${ }^{4}$ and F. Martín ${ }^{4,5}$ \\ ${ }^{1}$ Department of Physics, University of Windsor, 401 Sunset Avenue, Ontario, Canada, N9B 3P4 \\ ${ }^{2}$ Canadian Light Source, 101 Perimeter Road, Saskatoon, Saskatchewan, Canada, S7N 0X4 \\ ${ }^{3}$ Departamento Química Física I, Facultad de Ciencias Químicas, Universidad Complutense de Madrid, 28040 Madrid, Spain \\ ${ }^{4}$ Departamento de Química, Modulo 13, Universidad Autónoma de Madrid, 28049 Madrid, Spain \\ ${ }^{5}$ Instituto Madrileño de Estudios Avanzados en Nanociencia (IMDEA-Nanociencia), Cantoblanco, 28049 Madrid, Spain
}

(Received 2 August 2011; published 11 January 2012)

\begin{abstract}
Dissociative photoionization (DPI) of randomly oriented $\mathrm{H}_{2}$ molecules has been studied using linearly polarized synchrotron radiation at selected photon energies of 31,33 , and $35 \mathrm{eV}$. Large amplitude oscillations in the photoelectron asymmetry parameter $\beta$, as a function of electron energy, have been observed. The phase of these $\beta$ oscillations are in excellent agreement with the results of recent close coupling calculations [Fernández and Martín, New J. Phys. 11, 043020 (2009)]. We show that the oscillations are the signature of interferences between the $1 Q_{1}{ }^{1} \Sigma_{u}{ }^{+}$and $1 Q_{2}{ }^{1} \Pi_{u}$ doubly excited states decaying at different internuclear distances. The oscillations thus provide information about the classical paths followed by the nuclei. The presence of such oscillations is predicted to be a general phenomenon in DPI.
\end{abstract}

DOI: 10.1103/PhysRevLett.108.023004

PACS numbers: 33.80.Eh, 33.20.Ni, 33.60.+q

Photoionization dynamics of the smallest and most abundant molecule in the Universe, $\mathrm{H}_{2}$, continues to be a hot topic revealing new surprises on this most basic of processes. This has direct relevance in planetary atmospheres, interstellar clouds, and in plasma physics, and fundamental interest is fueled by increasingly more sophisticated theoretical descriptions (e.g., [1-4]) and advances in light sources and charged particle detection techniques, such as momentum imaging (e.g., [5-7]). In this study we consider the dissociative photoionization (DPI) process, $h \nu+\mathrm{H}_{2} \rightarrow \mathrm{H}+\mathrm{H}^{+}+e^{-}$, in which ionization and dissociation both occur on a very short time scale and the coupling between the electrons and nuclei can lead to the observation of interference phenomena $[4,8]$. In particular, we focus on the region between $h \nu=31-35 \mathrm{eV}$ where DPI can proceed directly or via doubly excited neutral states that promptly autoionize [9]. We observe large amplitude oscillations in the photoelectron asymmetry parameter $\beta$ as a function of electron energy resulting from the interference between autoionization decay pathways associated with specific doubly excited states. What is particularly dramatic is that such interference effects are prominently evident even in the case of randomly orientated $\mathrm{H}_{2}$ molecules.

The dominant ionization process results in bound vibrational levels of $\mathrm{H}_{2}^{+} X^{2} \Sigma_{g}^{+}\left(1 s \sigma_{g}\right)$-see Fig. 1. DPI can occur at $h \nu \geq 18.076 \mathrm{eV}$ resulting in $\mathrm{H}(1 s)+\mathrm{H}^{+}+e^{-}$ and at $h \nu \geq 28.281 \mathrm{eV}$ where the $\mathrm{H}(n=2)+\mathrm{H}^{+}+e^{-}$ channel opens. The next two ionic states of $\mathrm{H}_{2}{ }^{+}$, ${ }^{2} \Sigma_{u}^{+}\left(2 p \sigma_{u}\right)$ and ${ }^{2} \Pi_{g}\left(2 p \pi_{u}\right)$, are both repulsive and converging to these limits are two Rydberg series labeled $Q_{1}$ $\left(2 p \sigma_{u}, n l \lambda\right)$ and $Q_{2}\left(2 p \pi_{u}, n l \lambda\right)(n>1)$, respectively. These doubly excited states can autoionize to the bound ground state ion, $\mathrm{H}_{2}{ }^{+2} \Sigma_{g}^{+}\left(1 s \sigma_{g}\right)$, or dissociatively ionize, or produce neutral fragments. Listed below are competing DPI processes for $h \nu=31-35 \mathrm{eV}$, where both the $Q_{1}$ and $Q_{2}$ doubly excited states are accessible

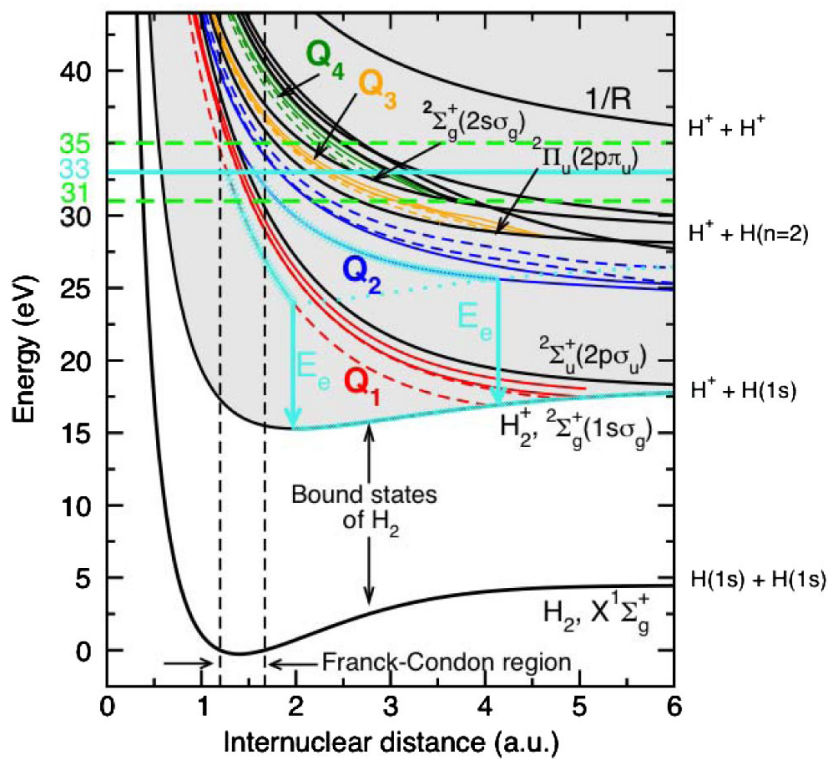

FIG. 1 (color online). The potential energy curves of the $\mathrm{H}_{2}$ and $\mathrm{H}_{2}{ }^{+}$systems from [4] with the shaded area representing the ionization continuum and the dashed vertical lines corresponding to the Franck Condon region from the ground vibrational level. Of particular interest to $h \nu=31-35 \mathrm{eV}$ energy region are families of $Q_{1}$ and $Q_{2}$ doubly excited states of ${ }^{1} \Pi_{u}$ and ${ }^{1} \Sigma_{u}^{+}$ symmetry designated by full and dashed curves, respectively. Illustrative traces are also shown to depict a representative semiclassical pathway to DPI via the lowest $Q_{1}$ and $Q_{2}$ states, resulting in electrons of identical energies. 


$$
\begin{aligned}
& h \nu+\mathrm{H}_{2}\left(X^{1} \Sigma_{g}^{+}\right) \rightarrow \mathrm{H}_{2}{ }^{+2} \Sigma_{g}^{+}\left(1 s \sigma_{g}\right)+e^{-} \rightarrow \mathrm{H}^{+} \\
& \quad+\mathrm{H}(1 s)+e^{-}, \\
& h \nu+\mathrm{H}_{2}\left(X^{1} \Sigma_{g}^{+}\right) \rightarrow \mathrm{H}_{2}{ }^{+2} \Sigma_{u}^{+}\left(2 p \sigma_{u}\right)+e^{-} \rightarrow \mathrm{H}^{+} \\
& \quad+\mathrm{H}(1 s)+e^{-},
\end{aligned}
$$

$$
\begin{aligned}
& h \nu+\mathrm{H}_{2}\left(X^{1} \Sigma_{g}^{+}\right) \rightarrow \mathrm{H}_{2}^{* *}\left(Q_{1}{ }^{1} \Sigma_{u}^{+},{ }^{1} \Pi_{u}\right) \rightarrow \mathrm{H}_{2}{ }^{+2} \Sigma_{g}^{+}\left(1 s \sigma_{g}\right) \\
& \quad+e^{-} \rightarrow \mathrm{H}^{+}+\mathrm{H}(1 s)+e^{-}
\end{aligned}
$$

$$
\begin{aligned}
& h \nu+\mathrm{H}_{2}\left(X^{1} \Sigma_{g}^{+}\right) \rightarrow \mathrm{H}_{2}^{* *}\left(Q_{2}{ }^{1} \Sigma_{u}^{+},{ }^{1} \Pi_{u}\right) \rightarrow \mathrm{H}_{2}{ }^{+2} \Sigma_{g}^{+}\left(1 s \sigma_{g}\right) \\
& \quad+e^{-} \rightarrow \mathrm{H}^{+}+\mathrm{H}(1 s)+e^{-},
\end{aligned}
$$

$$
\begin{aligned}
& h \nu+\mathrm{H}_{2}\left(X^{1} \Sigma_{g}^{+}\right) \rightarrow \mathrm{H}_{2}^{* *}\left(Q_{2}{ }^{1} \Sigma_{u}^{+},{ }^{1} \Pi_{u}\right) \rightarrow \mathrm{H}_{2}{ }^{2} \Sigma_{u}^{+}\left(2 p \sigma_{u}\right) \\
& +e^{-} \rightarrow \mathrm{H}^{+}+\mathrm{H}(1 s)+e^{-} .
\end{aligned}
$$

The energy difference between the $\mathrm{H}_{2}{ }^{+2} \Sigma_{g}^{+}\left(1 s \sigma_{g}\right)$ and ${ }^{2} \Sigma_{u}^{+}\left(2 p \sigma_{u}\right)$ states is $\sim 17 \mathrm{eV}$ in the Franck Condon region. Consequently, if $\mathrm{H}_{2}$ is nonresonantly ionized into these two final states via processes (1) and (2), then the emitted photoelectrons will have very different energies [(1) $>\sim 6 \mathrm{eV}$ and $(2)<\sim 6 \mathrm{eV}]$ and so would be readily distinguishable. Since process (5) decays to the same final state as that of (2), this mechanism essentially further contributes to the production of low energy electrons. In the case of resonant ionization processes (3) and (4), which also results in electron energies $>\sim 6 \mathrm{eV}$, the lowest $Q_{1}$ and $Q_{2}$ states have ${ }^{1} \Sigma_{u}^{+}$and ${ }^{1} \Pi_{u}$ symmetries, respectively, and both autoionize on a $<10 \mathrm{fs}$ time scale [9]. This delayed emission can result in autoionization occurring at significantly larger internuclear separation, $R$, i.e., outside the Franck Condon region. The ejected electron energies depend critically on the $R$ at the moment of autoionization and hence it is possible to have electrons of very similar energies due to different decay processes, giving rise to interference effects.

When using linearly polarized light, the emission of photoelectrons from a random distribution of atoms or molecules has a characteristic differential cross section that is expressed in terms of an asymmetry or $\beta$ parameter [10]:

$\frac{d \sigma}{d \Omega}=\frac{\sigma}{4 \pi}\left[1+\beta P_{2}(\cos \theta)\right]=\frac{\sigma}{4 \pi}\left[1+\frac{\beta}{2}\left(3 \cos ^{2} \theta-1\right)\right]$.

Here $\sigma$ is the photoionization cross section for a particular ionic state and $\theta$ is the angle between the polarization axis and the direction of the ejected electron. The variation of $\beta$ with photoelectron energy depends on the partial waves which contribute to the final channel and is therefore a sensitive probe of the photoionization dynamics. $\beta$ lies within the range $2>\beta>-1$, the limits corresponding to $\cos ^{2} \theta$ and $\sin ^{2} \theta$ distributions, respectively. In direct photoionization $\beta$ is generally slowly varying with photon (and hence photoelectron) energy. However, when photoionization occurs via experimentally indistinguishable routes, such as directly (1) and via intermediate neutral states (3) and (4), this can give rise to dramatic changes in both the partial cross section and the angular distribution of the photoelectrons as a function of photon or photoelectron energy. This is evident in Fig. 2, in which we show the first experimental observation of the $\beta$ variation, as a function of photoelectron energy, $E_{k}$, for three different photon energies in the DPI region.

The $\beta$ parameters in this DPI study of $\mathrm{H}_{2}$ were measured using an electrostatic toroidal photoelectron spectrometer, whose details are given elsewhere $[11,12]$. The spectrometer was oriented so that electrons emitted at $0^{\circ}$ and $90^{\circ}$ to the polarization axis were both included in the final energyresolved and angle-dispersed image. The experiments were performed on the Variable Line Spacing Plane Grating Monochromator (VLS PGM) undulator beam line at the Canadian Light Source [13]. The photon energy resolution was $\sim 10 \mathrm{meV}$ at $\sim 33 \mathrm{eV}$ and we take the Stokes $S_{1}$ parameter to be 0.98 . The angular resolution has previously been determined to be $\pm \approx 2.5^{\circ}$ from helium photodouble
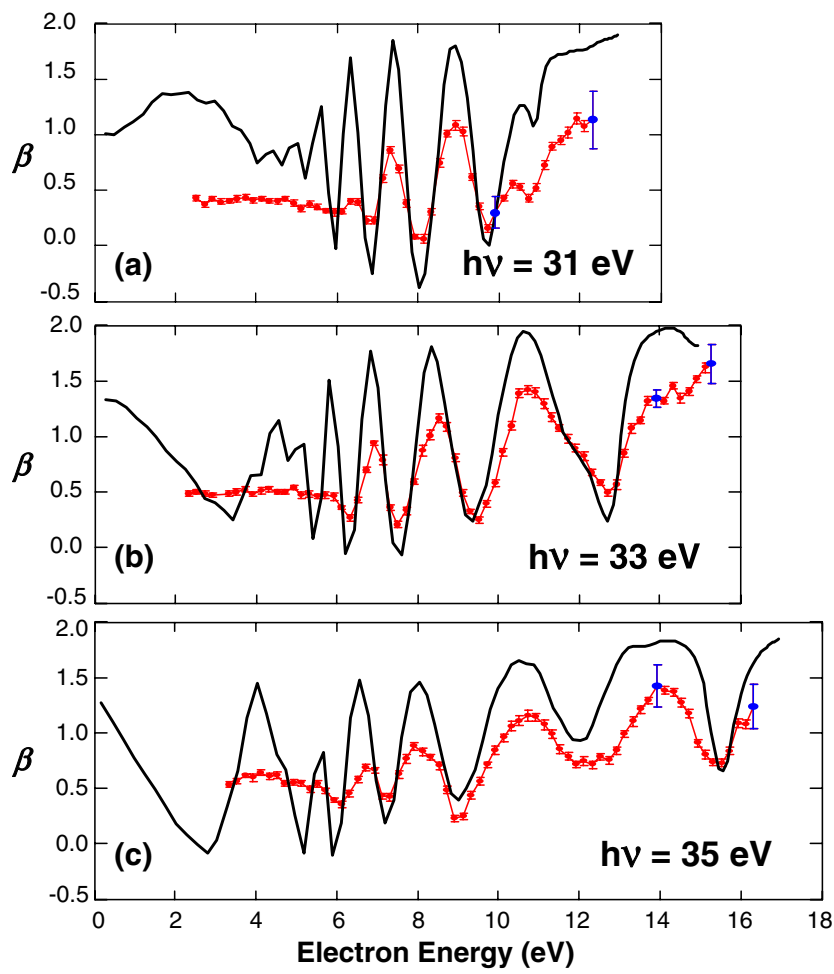

FIG. 2 (color online). Plot of the electron asymmetry parameter $(\beta)$ variation with electron energy for $h \nu=31,33$, and $35 \mathrm{eV}$; close coupling calculations (solid trace) and the measured data. The error bars on the calibration points at 9.9 and $13.9 \mathrm{eV}$ indicate the uncertainty in the overall $\beta$ scale; the relative statistical uncertainty is shown in the smaller error bars. The error bars for the highest photoelectron energies are a combination of the $\beta$ scale uncertainty and the statistical uncertainty associated with the sequential ratio fitting procedure. 
ionization studies [14]. The (angle-averaged) electron energy resolution, dominated by that of the toroidal analyzer, was measured as $\approx 100 \mathrm{meV}(\mathrm{FWHM})$ using $\mathrm{He}^{+}(n=2)$ photoelectrons.

The data acquisition and analysis used in this study are not how one typically measures $\beta$ parameters and full details will be presented elsewhere [15]. Briefly, at a given photon energy, the angle-dispersed photoelectron yield is recorded at each $E_{k}$ for a fixed number of counts (typically 25600 ). In this way the statistical quality of the data is uniform and the data acquisition time varies, depending on the photoionization cross section and experimental variables (photon flux, gas pressure, photon and electron resolutions). The recorded images are processed and the angular distributions are histogrammed in $5^{\circ}$ intervals. The variation in the detection efficiency with $\theta$ is remarkably stable for a particular spectrometer tuning. Variations can arise from, for example, local electric field irregularities, mechanical differences, and microchannel plate gain variations. However, by taking the ratio of two angular distributions obtained at very similar $E_{k}$ values (see below) one can eliminate the efficiency variation with emission angle and obtain a relatively smooth distribution that is proportional to the ratio of the differential cross sections.

The $\beta_{\mathrm{H}_{2}}$ spectra presented in Fig. 2 are placed on an absolute scale by performing a weighted least squares fit of the observed ratio of the $I_{\mathrm{H}_{2}}\left(\beta_{\mathrm{H}_{2}}, \theta\right) / I_{\mathrm{He}}\left(\beta_{\mathrm{He}}, \theta\right)$ yield using $\mathrm{He}(n=1, \beta=2)$ at selected photoelectron energies (see Fig. 2) using the same spectrometer tuning conditions at each $E_{k}$. Beginning with the above calibration point(s), the variation of $\beta_{\mathrm{H}_{2}}$ with $E_{k}$ is found by sequentially performing a weighted least squares fit of the observed ratio of the angular distribution, $I_{\mathrm{H}_{2}}$, obtained at $E_{k} \pm$ $0.2 \mathrm{eV}$ with that at $E_{k}$, i.e., of $I_{\mathrm{H}_{2}}\left(\left(E_{k} \pm \Delta E_{k}\right), \beta_{E_{k} \pm \Delta E_{k}}, \theta\right) /$ $I_{\mathrm{H}_{2}}\left(E_{k}, \beta_{E_{k}}, \theta\right)$ (where $\Delta E_{k}=0.2 \mathrm{eV}$ ), in order to determine $\beta_{E_{k} \pm \Delta E_{k}}$. The process is repeated successively throughout the spectrum, so that the fitted $\beta$ at $E_{k} \pm$ $0.2 \mathrm{eV}$ become the reference $\beta$ for the subsequent ratio (i.e., $\beta_{E_{k} \pm \Delta E_{k}} \rightarrow$ “ $\beta_{E_{k}}$ "). We assume that the spectrometer tuning conditions are essentially the same at each pair of photoelectron energies, since $\Delta E_{k}$ is small, i.e., a $\sim 2 \%$ change in $E_{k}$ at $E_{k} \sim 10 \mathrm{eV}$. Full details of the data analysis procedures, and a justification of the approximations involved, will be given in [15].

We have extended the calculations reported in Ref. [4] to the whole range of photon energies used in the present experiment. As can be seen, the comparison with theory reveals the presence of the recently predicted oscillations in $\beta$ as a function of $E_{k}$. Furthermore, there is a remarkable agreement in the phase of the oscillations at all three photon energies; the only minor exception being at $\sim 13 \mathrm{eV}$ in the $h \nu=35 \mathrm{eV}$ data. To place this experimental result in context, the landmark study of Lafosse et al [16], which examines DPI in the molecular frame, finds that $\beta \approx 1 \pm 0.1$ (for $0 \mathrm{eV} \leq E_{k} \leq 5 \mathrm{eV}$ ) and $\beta \approx 0.15 \pm 0.1$ (for $5 \mathrm{eV} \leq E_{k} \leq 10 \mathrm{eV}$ ) at $h \nu=32.5 \mathrm{eV}$. While the averaging over a broad $E_{k}$ is inherent in that coincidence technique, their findings are in very good agreement with the corresponding theoretical (average) $\beta$ 's of 1.16 and 0.2 , respectively.

There is, however, a general discrepancy in the amplitude of the oscillations in Fig. 2 which requires comment. First, the theoretical curve is not convoluted with the experimental photoelectron energy resolution, namely $\approx 100 \mathrm{meV}$ (FWHM). Incorporating this would reduce the amplitude of the oscillations. Second, and more importantly, there is a further experimental issue which systematically alters the absolute $\beta$ values below $E_{k} \sim 10 \mathrm{eV}$, namely the contribution due to low energy "background" (metal scattered) electrons. It is well known that energetic photoelectrons, in this case from the $\mathrm{H}_{2}{ }^{+2} \Sigma_{g}^{+}\left(1 s \sigma_{g}\right)$ ground state, undergo inelastic collisions with metal surfaces near the interaction region. The detected electron yield at a given $E_{k}$ will inevitably contain a background contribution from this photoinduced process and, unfortunately, the signal-tonoise ratio gets progressively worse as $E_{k} \rightarrow 0 \mathrm{eV}$. These background electrons are not isotropic, since they arise from photoelectrons with a high $\beta$, but their $\beta$ variation with $E_{k}$ has no structure. The effect of this increasing background contribution with decreasing $E_{k}$ is to suppress the amplitudes of the observed $\beta$ oscillations, but this does not alter the phase of the $\beta$ oscillations. This background electron issue will fade away rapidly for $E_{k}>\sim 10 \mathrm{eV}$ and therefore the reason for the observed discrepancy in the $\beta$ values is unclear in this $E_{k}$ region. We note that Hikosaka and Eland [17] also find $\beta$ values at $h \nu=21.2,23.1,26.9$, and $40.8 \mathrm{eV}$ that lie between $1.83 \rightarrow 1.69( \pm 0.05)$. Moreover, the vibrationally averaged (nondissociative) $\beta$ values for $h \nu=31,33$, and $35 \mathrm{eV}$ are approximately 1.9 , 1.75 , and 1.6, respectively [18], corresponding to the high $E_{k}$ "limit." There is, therefore, a body of experimental evidence that suggests $\beta$ at high $E_{k}$ values is significantly lower than $\beta=2.0$ of the united atom limit—helium—and of the $\mathrm{H}_{2}$ theoretical results presented in Fig. 2. Further work is needed to address this issue.

Returning to the $\beta$ oscillations themselves; is there a simple explanation as to how they arise? As demonstrated in Fig. 3(a) for $h \nu=33 \mathrm{eV}$, it is an excellent approximation to assume that only $\ell=1$ partial waves contribute to the ionization/autoionization process. Moreover, considering the ionization to be exclusively through the ${ }^{2} \Sigma_{g}^{+}\left(1 s \sigma_{g}\right)$ channel is a good approximation in the 5-15 eV region [4]. Thus, from Eq. (13) of Dehmer and Dill [10], we can write the electron $\beta$ as approximately

$$
\beta \approx \frac{2}{5\left(D_{p_{\sigma}}^{2}+2 D_{p_{\pi}}^{2}\right)}\left(2 D_{p_{\sigma}}^{2}+7 D_{p_{\pi}}^{2}+6\left|D_{p_{\sigma}}\right|\left|D_{p_{\pi}}\right| \cos \phi\right),
$$

where $D_{p_{\sigma}}$ and $D_{p_{\pi}}$ are the $\sigma$ and $\pi$ ionization amplitudes at a given electron kinetic energy $E_{k}$ and $\phi$ is the 


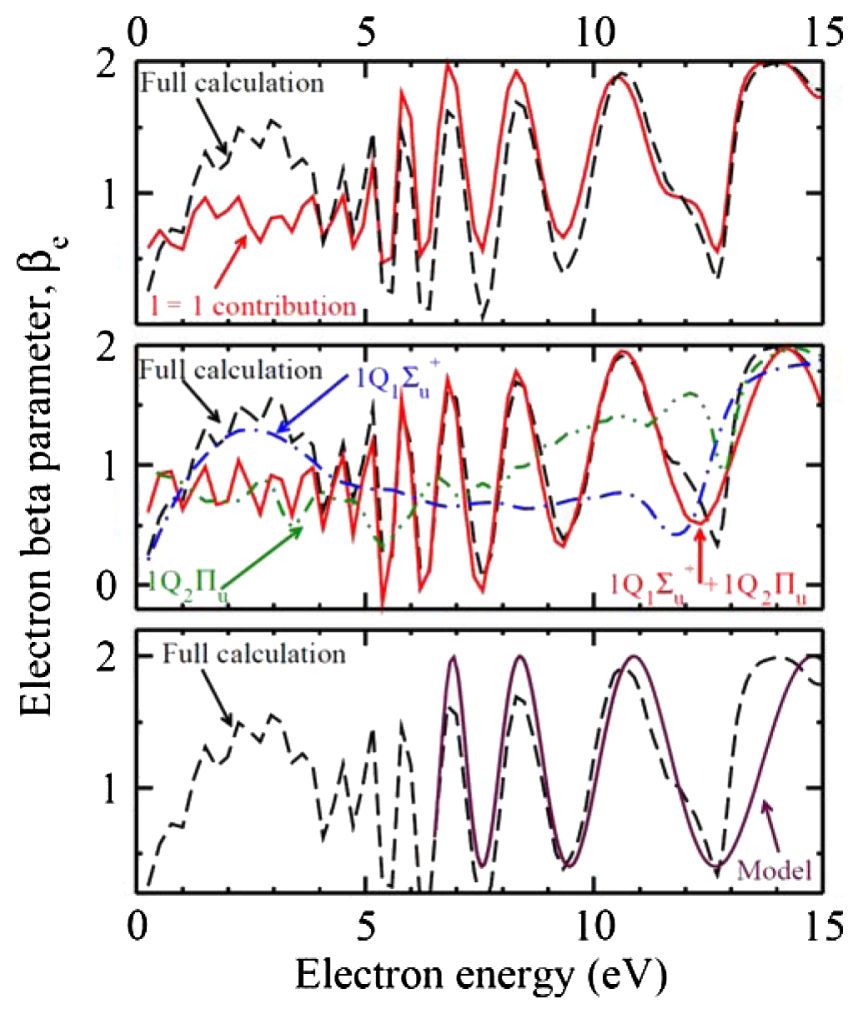

FIG. 3 (color online). Variation of the electron asymmetry parameter $\beta$, associated with the ${ }^{2} \Sigma_{g}^{+}\left(1 s \sigma_{g}\right)$ ionization channel with electron energy for $h \nu=33 \mathrm{eV}$. The black dashed curve is the result of our full $a b$ initio calculations. (a) Top panel shows the dominant $\ell=1$ partial wave contribution. (b) Middle panel shows the individual contributions of the $1 Q_{1}{ }^{1} \Sigma_{u}{ }^{+}$and $1 Q_{2}{ }^{1} \Pi_{u}$ amplitudes together with their coherent superposition, which gives rise to oscillations in $\beta$. (c) Bottom panel shows the results from the simple model presented in the text.

corresponding phase difference. Our ab initio calculations show that the above amplitudes are comparable in magnitude. For simplicity, we can assume that $\left|D_{p_{\sigma}}\right|=\left|D_{p_{\pi}}\right|$ for all energies so that

$$
\beta\left(E_{k}\right) \approx \frac{6}{5}+\frac{4}{5} \cos \left[\phi\left(E_{k}\right)\right] .
$$

From the calculations we also know that the largest contribution to the $D_{p_{\sigma}}$ and $D_{p_{\pi}}$ amplitudes comes from the $1 Q_{1}{ }^{1} \Sigma_{u}^{+}$and $1 Q_{2}{ }^{1} \Pi_{u}$ doubly excited states, respectively [see Fig. 3(b); notice also that these states do not lead to any oscillation when considered separately]. We now assume that $\phi_{i}$ is given by the difference between the phases accumulated along the two classical paths depicted in Fig. 1 plus an arbitrary energy-independent phase $\delta_{c}$. Within the semiclassical WKB approximation, the energydependent contribution is given by

$$
\begin{aligned}
\phi\left(E_{k}\right)= & \int_{R_{1}^{0}}^{R_{1}} d R k_{\mu}^{Q_{1}}(R)+\int_{R_{1}}^{R_{2}} d R k_{\mu}^{1 s \sigma_{g}}(R) \\
& -\int_{R_{2}^{0}}^{R_{2}} d R k_{\mu}^{Q_{2}}(R)
\end{aligned}
$$

where $R_{i}^{0}$ and $R_{i}$ are, respectively, the values of the internuclear distance at the beginning of the trajectory $[\omega=$ $\left.E_{Q_{i}}\left(R_{i}^{0}\right)\right]$ and at the point where the ejected electrons have energy $E_{k}, k_{\mu}^{N}(R)=\sqrt{2 \mu T_{N}(R)}$ is the nuclear momentum ( $\mu$ stands for the reduced mass of $\mathrm{H}_{2}{ }^{+}$), and $T_{N}(R)$ is the nuclear kinetic energy. For $R<R_{i}$, the latter is given by the difference between the photon energy and the energy of the $i$ autoionizing state at the internuclear distance $R$. For $R>$ $R_{i}, T_{N}(R)$ is given by the difference between the photon energy and the sum of the ground state ion and the outgoing electron energies. For the energy-independent part of the phase, we have chosen $\delta_{e} \sim \pi / 2$, whose only sizable effect is to shift the position of the maxima and minima of the cosine function. For every energy $E_{k}$, the $R_{i}^{0}$ and $R_{i}$ values are taken from our calculated curves for the $1 Q_{1}{ }^{1} \Sigma_{u}{ }^{+}$and $1 Q_{2}{ }^{1} \Pi_{u}$ doubly excited states, respectively (see Fig. 1). The results of such a model are shown in Fig. 3(c). As can be seen, the essence of the oscillatory behavior is reasonably caught. Indeed, the fact that the difference between $R_{1}$ and $R_{2}$ increases (decreases) with proton (electron) energy (see Fig. 1) leads to an energydependent frequency of the oscillations in reasonable agreement with the results of the $a b$ initio calculations. Therefore, the observed oscillations in the beta parameter as a function of electron energy are the signature of the interference between the $1 Q_{1}{ }^{1} \Sigma_{u}{ }^{+}$and $1 Q_{2}{ }^{1} \Pi_{u}$ doubly excited states decaying at different internuclear distances $R_{1}$ and $R_{2}$, and provide information about the different classical paths followed by the nuclei. We emphasize that the origin of the interference is the difference in phase between the two paths followed by the nuclear wave packets that result from autoionization of two distinct doubly excited states, while the interference observed in [8] is mainly due to the difference in the electronic phase associated with autoionization of a single doubly excited state into different final states. Finally, the small, systematic changes in the oscillations in Fig. 2 confirm that the same two states are responsible at all three photon energies.

This phenomenon will not be unique to $\mathrm{H}_{2}$. Similar oscillations in the beta parameter are expected whenever two autoionizing states decay at significantly different internuclear distances.

We acknowledge NSERC, CLS, and UoW for their support. We thank Mare Nostrum BSC and CCC-UAM for allocation of computer time. Work partially supported by the MICINN Projects No. FIS2010-15127, No. ACI2008-0777, and No. CSD 2007-00010, the European MC-ITN CORINF, and the COST Action CM0702.

[1] F. Martín, J. Phys. B 32, R197 (1999).

[2] W. Vanroose, D. A. Horner, F. Martín, T. N. Rescigno, and C. W. McCurdy, Phys. Rev. A 74, 052702 (2006). 
[3] J. Colgan, M. Foster, M. S. Pindzola, and F. Robicheaux, J. Phys. B 40, 4391 (2007).

[4] J. Fernández and F. Martín, New J. Phys. 11, 043020 (2009).

[5] R. Dörner, V. Mergel, O. Jagutzki, L. Spielberger, J. Ullrich, R. Moshammer, and H. Schmidt-Böcking, Phys. Rep. 330, 95 (2000).

[6] M. Lebech, J.C. Houver, and D. Dowek, Rev. Sci. Instrum. 73, 1866 (2002).

[7] M. Gisselbrecht, A. Huetz, M. Lavollée, T. J. Reddish, and D.P. Seccombe, Rev. Sci. Instrum. 76, 013105 (2005).

[8] F. Martín, J. Fernández, T. Havermeier, L. Foucar, Th. Weber, K. Kreidi, M. Schöffler, L. Schmidt, T. Jahnke, O. Jagutzki, A. Czasch, E. P. Benis, T. Osipov, A. L. Landers, A. Belkacem, M.H. Prior, H. Schmidt-Böcking, C. L. Cocke, and R. Dörner, Science 315, 629 (2007).

[9] I. Sánchez and F. Martín, J. Chem. Phys. 106, 7720 (1997); 110, 6702 (1999).

[10] J. L. Dehmer and D. Dill, Phys. Rev. A 18, 164 (1978).
[11] T. J. Reddish, G. Richmond, G. W. Bagley, P. J. Wightman, and S. Cvjanović, Rev. Sci. Instrum. 68, 2685 (1997).

[12] J.P. Wightman, S. Cvejanović, and T. J Reddish, J. Electron Spectrosc. Relat. Phenom. 95, 203 (1998).

[13] Y. F. Hu, L. Zuin, G. Wright, R. Igarashi, M. McKibben, T. Wilson, S. Y. Chen, T. Johnson, D. Maxwell, B. W. Yates, T. K. Sham, and R. Reininger, Rev. Sci. Instrum. 78, 083109 (2007).

[14] J. P. Wightman, S. Cvejanović, and T. J Reddish, J. Phys. B 31, 1753 (1998).

[15] M. A. MacDonald et al. (to be published).

[16] A. Lafosse, M. Lebech, J. C. Brenot, P. M. Guyon, L. Spielberger, O. Jagutzki, J. C. Houver, and D. Dowek, J. Phys. B 36, 4683 (2003).

[17] Y. Hikosaka and J.H.D. Eland, J. Electron Spectrosc. Relat. Phenom. 133, 77 (2003).

[18] A. C. Parr, J. E. Hardis, S. H. Southworth, C. H. Feigerle, T. A. Ferrett, D. M. P. Holland, F. M. Quinn, B. R. Dobson, J. B. West, G. V. Marr, and J. L. Dehmer, Phys. Rev. A 37, 437 (1988). 